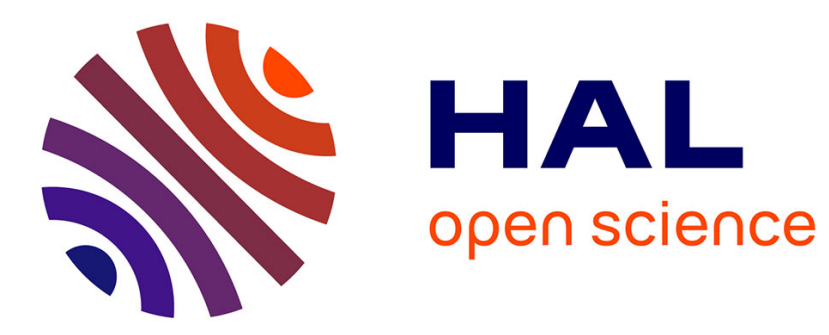

\title{
Le Parti communiste et le service public
}

Dominique Andolfatto

\section{To cite this version:}

Dominique Andolfatto. Le Parti communiste et le service public. Emmanuel Cherrier; Stéphane François. Le service public et les idéologies politiques, Presses Universitaires du Septentrion, pp.4761, 2016, Les Savoirs mieux, 978-2-7574-1380-7. hal-01696676

\section{HAL Id: hal-01696676 https://hal.science/hal-01696676}

Submitted on 24 Nov 2021

HAL is a multi-disciplinary open access archive for the deposit and dissemination of scientific research documents, whether they are published or not. The documents may come from teaching and research institutions in France or abroad, or from public or private research centers.
L'archive ouverte pluridisciplinaire HAL, est destinée au dépôt et à la diffusion de documents scientifiques de niveau recherche, publiés ou non, émanant des établissements d'enseignement et de recherche français ou étrangers, des laboratoires publics ou privés. 
Dominique Andolfatto

Professeur de science politique

Credespo, Université de Bourgogne

Dominique.Andolfatto@u-bourgogne.fr

\section{Le Parti communiste et le service public}

Texte publié dans : Emmanuel Cherrier, Stéphane François, dir., Le service public et les idéologies politiques, Lille, Septentrion Presses Universitaires, coll. Les savoirs mieux, 2016, p. 47-61.

Comment définir la notion de service public ? Elle renvoie habituellement à deux éléments : une mission correspondant à une activité d'intérêt général; un certain mode d'organisation de cette activité, soit sa prise en charge directe ou indirecte par un organisme public (Etat, collectivités territoriales ou établissements publics) voire par une entreprise privée mais sous contrôle public. La notion a nourri bien des débats, suscité bien des actions collectives. On citera, à titre d'exemple, les grèves de l'automne 1995, marquées par une importante «mobilisation » de fonctionnaires ou d'agents de services publics - transports, poste, énergie... - contre une certaine conception de la «modernisation» des services publics, inspirée du new public management, et de la remise en cause d'acquis sociaux, tels les régimes spéciaux de retraite... C'est d'ailleurs cette "modernisation », quasi-permanente depuis au moins les travaux de Michel Crozier sur Le phénomène bureaucratique et le volontarisme gaulliste concernant la restauration et l'efficacité de l'Etat, qui cristallise prises de position, actions collectives, voire «résistances» de la part de syndicats ou partis politiques, ces derniers adoptant le plus souvent des positions «défensives » face à des réformes supposées remettre en cause, sinon démanteler, les services publics.

Un autre exemple, plus récent de ces «mobilisations» serait - le 9 octobre 2009 l'organisation d'une « votation citoyenne sur la privatisation de la poste » (selon l'expression des ses promoteurs). Il s'agissait d'exiger, au-delà de ce vote, un référendum national pour défendre le «service public postal. Le PCF, le NPA, ainsi que tout l'échiquier syndical, se trouvaient ici à la manœuvre. La non reconnaissance par l'Union européenne des services publics « à la française », l'exigence d'ouverture à la concurrence de certains d'entre eux ont nourri également l'action collective.

Comment se positionne un parti tel le PCF dans cette problématique défensive des services publics ? Plus au fond, existe-t-il une idéologie «communiste» du service public ? Cela renvoie en fait à une question préalable concernant l'idéologie communiste aujourd'hui. En effet, depuis la chute du mur de Berlin, et des régimes qui s'étaient imposés à l'ombre de celui-ci, le PCF ne développe plus un discours idéologique caractéristique. Il fait montre de réserve et de prudence. Sur bien des sujets, il paraît même s'être converti à la «socialdémocratie », sans jamais toutefois la nommer, car, pour des raisons tenant à l'identité profonde du parti, ce positionnement reste rejeté sinon considéré comme hérétique. Il reste que le PCF n'a pas complètement renoncé aux « lendemains qui chantent ». Mais le discours communiste a changé et la direction du parti préfère désormais - publiquement - les poètes 
aux idéologues, plaçant par exemple le 36 ime congrès du parti, en février 2013, sous l'égide d'un vers d'Apollinaire : il est « grand temps de rallumer les étoiles »'.

Cela dit, la notion de communisme, telle que formulée lors de ce congrès, demeure assez vague, tant dans ce qui serait les promesses de celui-ci que dans le processus qui permettrait de les réaliser. Le texte adopté lors de ce congrès indique en l'occurrence que le PCF porte un projet qui doit se substituer au capitalisme en raison d'une contradiction «de plus en plus insoutenable » entre ce dernier et le «progrès social », la « démocratie », le « développement culturel », l' «écologie » et la «paix ». La nécessité d'une révolution se trouve également reformulée. Mais il ne s'agira plus une "prise de pouvoir d'une minorité », soit d'un coup de force ou de ce qui serait un «nouvel octobre ». Cette révolution - selon le texte adopté lors du $36^{\text {ine }}$ congrès du PCF - sera «sociale, citoyenne, pacifique, démocratique ». Elle traduira l'avènement d'un «communisme de nouvelle génération $»^{2}$, communisme non seulement incompatible avec l'austérité mais consistant en un "incessant mouvement démocratique d'appropriation citoyenne du monde et de partage des avoirs, des savoirs et des pouvoirs qui enverra aux oubliettes l'ancien régime du capitalisme et fera grandir l'humain dans l'humanité $»^{3}$. Cet extrait pourrait donner l'impression d'un exercice purement rhétorique mêlant allitérations et assonances mais, finalement, peu éclairant sur le fond. Le texte du $36^{\mathrm{me}}$ congrès mentionne encore que le communisme est une "visée », soit un projet de société "débarrassée de toute forme d'aliénation, d'exploitation, de domination, de prédation de la planète $»^{4}$. Au total, ce texte renseigne assez peu sur le communisme (ou néo-communisme), sauf à se démarquer « de l'affrontement qui [s'est joué] dans la guerre froide » (seule allusion au «passé d'une illusion » pour reprendre une célèbre expression de François Furet) et à ajouter une dimension écologique à une dimension socio-économique plus traditionnelle. On repère toutefois, à de nombreuses reprises, l'évocation du service public (au singulier ou au pluriel).

C'est en prenant appui sur ces nombreuses occurrences - à travers un dépouillement systématique du journal hebdomadaire Communistes. Lien d'échanges et de communication (qui est un supplément hebdomadaire de L'Humanité) - que le développement qui suit est construit. En effet, il n'est guère de semaines sans que ce journal ne parle du ou des service(s) public(s). Bien plus, il multiplie parfois leur évocation alors que le journal n'a qu'un format court (c'est le plus souvent un « 4 pages »). Son dépouillement, de l'automne 2012 (dans le contexte de préparation du $36^{\text {ime }}$ congrès du PCF) au printemps 2014 (le contexte étant alors celui de la campagne municipale) - soit 54 numéros -, permet de recenser 143 références $\mathrm{au}(\mathrm{x})$ service(s) public(s), soit près de 3 références en moyenne par livraison de Communistes. Notons aussi que la livraison spéciale, relative au compte rendu du $36^{\text {ine }}$ congrès du PCF, mentionne 38 fois (en 24 pages) le (ou les) service(s) public(s). Et le « texte d'orientation » adopté lors de ce congrès (on parlait autrefois de la «thèse » ou de la « résolution » du congrès) reprend à 22 reprises la même expression (en 41 pages).

Ce dépouillement de l'hebdomadaire des communistes a été complété par la lecture - ou relecture - d'ouvrages de quelques dirigeants communistes afin de réintroduire de la profondeur dans l'étude et l'analyse et de mesurer des évolutions éventuelles dans le recours et les usages de la notion de service public. Ces livres sont signés par Georges Marchais, ancien secrétaire général du PCF (1972-1996), Pierre Blotin, qui inspira la «mutation » à la fin des années 1990, Marie-George Buffet, ancienne secrétaire nationale au début des années 2000, Anicet Le Pors, qui fut l'un des quatre ministres communistes en 1981, chargé de la

Voir: PCF, Humanifeste du Parti communiste français à l'aube du siècle qui vient, Texte d'orientation adopté par le 36 congrès du PCF, 10 février 2013.

Ibid., p. 3.

Ibid., p. 1 et 16.

Ibid., p. 17. 
fonction publique et des réformes administratives. Certains propos sur l'Etat de Maurice Thorez, autre ancien secrétaire général du PCF (1930-1964), sont également extraits d'un livre d'Yves Roucaute (1979). Ce choix pourra paraître aléatoire mais il s'agit de constituer un groupe d'auteurs de référence du communisme français pour tenter de déterminer quel usage - depuis une quarantaine d'années - le Parti communiste a fait du service public.

\section{Une apologie du service public}

Le dépouillement de Communistes conduit à repérer quatre approches de la notion de service public :

- la défense d'une notion étonnamment indéfinie (et présentant du coup une dimension quasi-mystique) ;

- une vision très large du service public (tout ou presque en relève, au moins potentiellement) ;

- le service public comme substitut à des termes plus anciens, propres au discours communiste, mais qui n'ont plus cours (tel celui de « collectivisation»);

- enfin, une approche du service public en tant qu'instrument économique.

\section{Défendre l'indéfini}

Les contributeurs de Communistes se révèlent d'abord en défenseurs infatigables des (ou du) services publics, sans que ceux-ci soient précisément définis. Cela se fait à travers la défense d'une notion qui serait naturellement menacée. Ainsi, dans Communistes sont dénoncés :

- la « dégradation constante des services publics » ( $n^{\circ}$ 519, 29 mai 2013);

- leur « sacrifice » en raison des politiques « austéritaires » $\left(\mathrm{n}^{\circ} 518,22\right.$ mai 2013) ;

- la « disparition des services publics » $\left(n^{\circ} 507,13\right.$ février 2013);

- la « destruction » des services publics (id.).

Dans la même veine, la direction du PCF affiche pour slogan «stop à la casse des services publics $\gg\left(n^{\circ} 511\right.$ et 513, 20 mars et 3 avril 2013).

A ces dénonciations et craintes pour l'avenir proche du service public s'ajoute - autre récurrence - la nécessité à l'inverse de développer les services publics. Là aussi, le discours est récurrent et faiblement argumenté. Ce développement des services publics qui, le plus souvent, va de soi, peut être ponctuellement justifié par de brèves argumentations de type social, économique, voire psychologique. Ainsi, le développement des services publics doit « répondre aux nouveaux besoins de la population» ( $\mathrm{n}^{\circ} 507,13$ février 2013). Il doit répondre également aux «besoins accrus de la population» ( $\mathrm{n}^{\circ} 498,28$ novembre 2012). Mais ces «besoins » ne sont pas déclinés si ce n'est à une reprise au cours de la période dépouillée : il s'agit d' "aider au quotidien les privés d'emploi ». Cependant aucun service particulier ou nouveau n'est ici précisé (n 534, 20 novembre 2013).

Renforcer les services publics doit aussi permettre également de «développer les capacités humaines » mais cet aspect, assez vague, n'est pas non plus explicité.

L'argumentation peut conduire à souligner enfin la dimension économique des services publics. Leur « développement» constitue «un éléments essentiel pour sortir de la crise » (512). Ils constituent encore une «alternative à l'austérité » ( ${ }^{\circ} 508,20$ février 2013) comme cela sera précisé ci-dessous.

$\mathrm{Au}$ total, le contenu de la notion de service public apparaît généralement vague, trop souvent indéfini. Le registre paraît d'abord incantatoire. 


\section{Tout est-il service public?}

Le dépouillement de Communistes permet tout de même de lister plus précisément à quoi - services ou missions - renvoie la notion de service public selon la direction du PCF. Une liste assez classique - et attendue - peut être dressée mais elle n'est pas sans réserver quelques surprises.

Au niveau local, la notion s'applique aux secteurs de la santé, l'éducation, le logement, la petite enfance $\left(\mathrm{n}^{\circ}\right.$ 518, 22 mai 2013). Dans le contexte de la campagne des élections municipales de 2014, certaines fédérations disent organiser des manifestations en faveur de ces services publics, comme dans le Lot-et-Garonne. A plusieurs reprises, l'hebdo Communistes associe d'ailleurs les services publics à la notion de proximité, qui connaît une certaine mode depuis quelques années, redonnant du sens au lien social et, ici, légitimant ou re-légitimant certaines institutions publiques. Ainsi, le service public serait d'abord une institution proche des habitants.

Cette proximité permettrait d'assurer l'égal accès de tous à tel service. Cela faciliterait aussi un contrôle citoyen (dont les modalités restent toutefois à définir). En outre, cette importance accordée à la «localisation» des services publics conduit a contrario à une critique de l'acte III de la décentralisation (qui a abouti - provisoirement - à la loi «métropole» du 27 janvier 2014). Or cette loi est rejetée parce que substituant «aux principes républicains de solidarité et d'égalité des territoires une concurrence exacerbée entre les territoires et les hommes au seul profit des grands groupes financiers de taille mondiale » $\left(n^{\circ} 542,29\right.$ janvier 2014). Et de poursuivre après ce raccourci : «cela aura des conséquences dramatiques sur l'emploi, sur la qualité et l'accessibilité des services publics. » L'analyse paraîtra assez dogmatique et définitive. Elle n'est pas non plus très explicite sauf dans une livraison de Communistes indiquant que le service public est «essentiel à l'aménagement équilibré [des] territoires » $\left(\mathrm{n}^{\circ} 532,23\right.$ octobre 2013$)$.

Spécifiquement, le service public concerne aussi la gestion de l'eau. Compte tenu d'une «situation dégradée », la direction du PCF se prononce pour la mise en place d'un service public national de l'eau ( $\mathrm{n}^{\circ}$ 532, 23 octobre 2013). Plus largement, il s'agit aussi de défendre le «service public ferroviaire ». Une brochure relative à celui-ci a d'ailleurs été publiée (en lien avec la «réforme ferroviaire» lancée en 2013)s. Pour garantir le «service public ferroviaire », il importe - selon la direction du PCF - de redonner plus de force à l'Etat au sein de la SNCF. Il s'agit aussi de libérer la SNCF de la concurrence (sans qu'il soit question explicitement d'un retour à un monopole ou à l'étatisation). Il importe également de garantir le statut des cheminots présenté comme un « contrat social » liant chaque cheminot au service public ferroviaire.

Autre champ naturel des services publics qui doit prendre un «nouvel essor»: l'Education nationale ( $\mathrm{n}^{\circ}$ 526, 11 septembre 2013). De même la recherche, la culture, la protection de l'environnement ( $\mathrm{n}^{\circ}$ 507, 13 février 2013). Enfin, la direction du PCF défend les « services publics forestiers » pour lutter contre la déforestation. La liste paraîtra finalement indéfinie, presque «à la Prévert». Tout ce qui est «bien commun» ou concerne le développement des individus aurait naturellement vocation à constituer un service public et à être défendu comme tel.

Enfin, consécutivement à l'accord national interprofessionnel sur la compétitivité et la sécurisation de janvier 2013, la direction du PCF prône également le développement d'un « service public de l'orientation et de l'insertion professionnelle » $\left(\mathrm{n}^{\circ}\right.$ 508, 20 février 2013), à distinguer a priori de Pôle Emploi. Il est également question d'un « service public d'emploi et de formation » où chacun pourra s'affilier dès la fin de sa scolarité $\left(\mathrm{n}^{\circ} 495,7\right.$ novembre

\footnotetext{
Système ferroviaire. Politique des transports, brochure du PCF et du Front de gauche, 2013.
} 
2012). Cela constituerait une innovation assurant le lien entre la formation et l'emploi. On pourrait y voir les débuts d'une sécurité sociale professionnelle pour faire écho à une revendication de la CGT. Cela étant, l'intégration dans l'emploi serait complètement « socialisé », prenant appui sur divers services publics.

Bien plus, dans un rapport présenté au conseil national (ex-comité central) du PCF, l'un de ses dirigeants, Olivier Dartigolles, souhaite même la mise en place d'un «service public des économistes qui veulent servir l'économie et désarmer la finance » ( $\mathrm{n}^{\circ}$ 498, 21 novembre 2012). Autrement dit, le service public servirait une économie étroitement dirigée en même temps que la société serait encadrée par toutes une séries d'institutions collectives assurant insertion professionnelle et bien-être.

Enfin, la direction du PCF appelle de ses vœux la mise en place d'un «pôle d'entreprises », avec mission de service public, pour gérer et rationaliser les aides à la presse... Sans doute, s'agit de garantir la pérennité de L'Humanité mais aussi celle d'autres médias. Plus globalement, c'est donc une cohorte d'institutions qui «garantirait » l'économie, la cohésion sociale et même l'opinion...

La liste des services publics - à la lecture de Communistes - paraîtra finalement inépuisable. Tout ce qui est «bien commun » ou concerne le développement social, culturel, éducatif, économique, voire personnel, a naturellement vocation à constituer un service public et à être défendu comme tel. Il reste que le rôle ou les missions de ces institutions, comme leur mode de fonctionnement ou de gouvernance, ne sont pas discutés, sauf à souligner ponctuellement la nécessité de restaurer le rôle de l'Etat ou de renforcer celui des élus. Si un certain rôle incombe également aux citoyens, comme cela sera précisé ci-dessous, tout cela traduit aussi une vision de la société civile qui ne saurait être autonome de tout un cortège institutionnel

\section{Une philosophie politique du service public?}

Il se dégage également de la lecture de Communistes une certaine philosophie du service public. On peut au passage se demander si cette expression ne serait pas un substitut à des termes plus en anciens - «collectivisme » ou «collectivisation », « étatisation », «prise de contrôle », «nationalisation »... - qui ne sont plus en vogue et ont disparu le plus souvent du discours communiste contemporain. Le service public serait-il la forme euphémisée d'un communisme (ou plutôt d'une collectivisation) qui n'oserait plus dire son nom ?

Si l'on suit l'argumentation de Pierre Laurent, le secrétaire national du parti, lors du $36^{\text {ime }}$ congrès de celui-ci, en 2013, les services publics constituent une valeur commune associé au «partage », à la «coopération», au «bien commun» et ils s'opposeraient, en termes inversés, aux maux ou aux vices du capital... Ce serait bien là une manière de réinventer une doctrine communiste. Le «manifeste» adopté lors du même congrès propose par ailleurs « une administration démocratisée des services publics et leur expansion coopérative jusqu'à la gestion des biens communs de l'humanité ». Il y a bien là l'idée d'une «collectivisation» progressive de certains biens (qui n'est pas sans rappeler Louis Blanc et son « organisation du travail», sorte de pré-marxisme), mais cette «collectivisation» se ferait sous contrôle citoyen.

Selon la fondation Rosa Luxemburg, proche du parti allemand Die Linke - et indirectement du PCF et du Front de gauche - «les «biens communs » font référence aux «éléments indispensables à la vie, comme l'eau et les semences » ainsi qu'aux « services publics », aujourd'hui démantelés dans bien des pays ${ }^{6}$. Mais ces «biens » doivent être vus

'François Houtard, Des biens communs au bien commun de l'humanité, Bruxelles, Fondation Rosa Luxemburg, 2011, p. 7. 
aussi - selon la même fondation - comme un «instrument de travail concret » face aux crises : il s'agit en l'occurrence de faire converger «des résistances et des luttes contre un système destructeur de la nature et des sociétés ». C'est aussi, très concrètement, prendre appui sur - et développer - «la solidarité (...) l'altruisme, le respect de la nature, de la tendresse, bref de tout ce qui constitue l'humain $»^{\prime}$.

Ainsi, le service public - pour revenir à lui - revêt à la fois une dimension sectorielle, instrumentale et même psychologique. Il protège, épanouit et permet aussi de lutter. Ce faisant, il ressemblerait à une institution magique : il répondrait à nombre de besoins, résoudrait nombre de difficultés, favoriserait le développement personnel. Pour le PCF, il traduit aussi une conception de la démocratie. C'est même «une exigence de démocratie » peut-on lire dans Communistes ( $\mathrm{n}^{\circ} 530,9$ octobre 2013). Il représente en l'occurrence une certaine forme de démocratie participative - ou mixte - telle que la conçoit le PCF. Concernant cet aspect, le manifeste adopté par le PCF lors de son 36 ime congrès mentionne : «Les agents des services publics et leurs usagers doivent disposer de droits et pouvoirs nouveaux pour leur permettre de participer à la définition même des missions à remplir, à celle des moyens institutionnels et financiers à mobiliser, à l'évaluation et au contrôle des services publics... » $\left(\mathrm{n}^{\circ}\right.$ 507, 13 février 2013).

Enfin le service public paraît recouvrir une dimension sociologique. Il constitue littéralement une sorte de catégorie sociale, au même titre, qu'autrefois, la mythique classe ouvrière. Ainsi, symboliquement, lors de l'ouverture du $36^{\text {ime }}$ congrès du PCF, un accueil particulier est réservé aux « délégations de salariés en lutte » et, au côté des représentants de nombreuses entreprises (Florange, Virgin, Alcatel...), qui ont polarisé l'actualité sociale en amont du congrès, on trouve des « représentants du service public » $\left(\mathrm{n}^{\circ} 507,13\right.$ février 2013). Ainsi, l'agent des services publics apparaît comme l'archétype du nouveau lutteur. Il assure la relève des ouvriers et perpétue le combat de classe.

\section{Le service public, instrument économique}

Comme déjà souligné, l'hebdo Communistes souligne également l'intérêt utiliser les services publics «pour soutenir [une économie de] l'offre et la demande à la fois » $\left(\mathrm{n}^{\circ} 543\right.$, 5 février 2014). On y verra une critique des inflexions de la politique économique de François Hollande. Mais l'analyse prend en compte aussi le niveau européen. Le PCF y défend un développement des services publics alors que ceux-ci sont très discutés à Bruxelles. Là aussi, il s'agit donc de renverser la table.

Selon l'un des économiste du PCF, Yves Dimicoli, cet impératif doit prendre appui sur un «fonds social, solidaire et écologique de développement européen » qui - c'est un peu complexe - « recueillerait la monnaie créée par la BCE à l'occasion des achats de titres publics émis par chaque pays pour le financement de ses services publics » ( $\mathrm{n}^{\circ} 543,5$ février 2014). En l'occurrence, ce fonds « redistribuerait cette monnaie à chaque Etat selon les besoins sociaux et culturels propres de son peuple » mais aussi en fonction de critères démocratiques. Tout cela mériterait évidemment d'être plus explicite. En d'autres termes, de «bonnes pratiques » démocratiques - mais évaluées comment ? - et aussi une économie des besoins - mais, là encore, en fonction de quels critères - justifieraient le financement par l'Europe de services publics. En outre, ces derniers ne sont évoqués que comme une notion générique et leur impact économique ne fait pas l'objet d'une analyse détaillée.

Lors du $36^{\text {im }}$ congrès du PCF, Yves Dimicoli réitérait l' « «exigence d'une vive expansion de tous les services publics que la BCE doit financer par sa création monétaire » ( ${ }^{\circ}$ 507, 13 février 2013). S'agit-il simplement de faire tourner la planche à billets et de voir

Ibid., p. 8. 
les services publics, et leur développement, comme une solution magique à la récession ? Cela cède manifestement à un certain simplisme sinon populisme - soit à une vision sommaire de la réalité - qui instrumentalise le service public en faisant de celui-ci un outil de lutte de catégories dominées face aux élites. L'hebdo Communistes préconise aussi - et plus clairement - l'instauration d'une taxe Tobin pour contribuer au financement des services publics en Europe ( $\mathrm{n}^{\circ}$ 521, 12 juin 2013).

\section{Un usage plus discret de la notion de service public par les élites communistes}

Tandis que la lecture de l'hebdo Communistes éclaire le discours communiste contemporain et tend à faire du service public une notion incontournable, voire centrale, de celui-ci, revisiter les écrits de dirigeants communistes du passé, même récent, et situer la notion de service public dans une perspective plus longue, témoignent d'une moindre centralité de celle-ci. Pour autant, elle apparaît souvent comme une clé pour le développement à tous niveaux.

Pierre Blotin, par exemple, qui fut l'un des mentors de Robert Hue, et inspira la «mutation » du PCF à la fin des années 1990, privilégie dans son livre sur le Communisme français (2006) le registre de la dénonciation. Il déplore la soumission des services publics aux marchés financiers. Dès lors les Etats ne peuvent plus faire face aux défis du chômage, de la pauvreté, de la ségrégation ethnique et ils ne peuvent plus, non plus, faire face à leurs obligations en matière de santé publique, de logement, d'aide à la création, de développement de grands projetss. Resterait à se demander si la notion de service public peut répondre à tous ces défis. Lutter contre le chômage ou la pauvreté n'est-il que l'affaire de services publics ?

Marie-George Buffet, dans Un peu de courage ! (2004), aborde le service public à travers - dit-elle - ce qu'on appelé «pudiquement l'ouverture du capital des entreprises publiques » (comme pour EDF en 2004). Dès lors, le service public ne serait plus assuré. Or, selon MarieGeorges Buffet, l'électricité, le gaz, les routes, les hôpitaux, les transports en commun, l'école... relèvent de la « responsabilité de l'Etat envers les citoyens ».

Dans ces conditions, seul le service public est à même d'assurer une égalité d'accès, une continuité du service, un « prix « échappant à la spéculation financière »".

Cependant - encore une fois - il ne s'agit là que de postulats, d'une sorte de croyance qui mériterait d'être étayée. Ainsi, les études sur la performance comparée de l'école sont particulièrement cruelles - comme on le sait - pour le système scolaire français ${ }^{10}$. De même, la continuité des services des transports semble mieux assurée dans d'autres pays qu'en France bien que ces derniers ne partagent pas la conception française du service public.

Marie-George Buffet souligne également l'importance de la présence de «services publics » pour la politique des quartiers". Mais, globalement, tout comme P. Blotin, elle recourt assez peu la notion de service public. Sauf à dénoncer les transformations juridiques qui ont affecté quelques entreprises, la notion semble assez étrangère à sa culture politique et à son discours. Cela tendrait bien à démontrer un usage et maniement relativement récent de celle-ci par les élites du parti.

Relire les livres signés par Georges Marchais montre de même que celui-ci évoquait peu la notion de services publics, longtemps confinée dans un registre strictement juridique. Ainsi,

\footnotetext{
P. Blotin (2006, p. 52).

M.-G. Buffet (2006, p. 20).

10 Voir les enquêtes PISA publiées par l'OCDE.

Ibid., p. 81.
} 
dans L'espoir au présent, publié en 1980, dans la perspective de l'élection présidentielle de 1981, il n'utilise pas cette notion tout en parlant tout de même du fond à laquelle renvoie le service public. Il évoque par exemple l'hôpital, déplorant toutefois - et déjà - «la vision étroitement comptable de ceux qui nous gouvernent $\gg^{2}$. Bref, le fond du propos résonne avec le discours contemporain, mais la notion de service public - en tant que telle - est absente.

G. Marchais parle également de l'école. Il met l'accent sur «le drame [de] ces établissements où les jeunes s'ennuient » et appelle de ses vœux « une école différente », une école où l'apprentissage ne repose pas seulement sur la «forme livresque ». La critique paraît ici plus développée et même plus incisive que celle du PCF d'aujourd'hui qui a comme sanctuarisé tout ce qui concerne l'école, sauf à dénoncer globalement les «menaces » qui pèseraient sur elle. Le livre critique également la «bureaucratie $» .$. avec ses «kilos de paperasse... cachets, tampons, timbres... » Cela contribue - argumente-t-il - à « saboter les services publics» que «L'Etat doit développer en les rendant plus démocratiques, plus accessibles à tous $\gg^{13}$. Il importe surtout d'améliorer les relations entre administrations et administrés, critique qui a semble-t-il disparu aujourd'hui, Etat, administrations, services publics échappant à ce type de mise en cause dans le discours communiste, la critique s'étant donc déportée. De même, des termes comme «bureaucratie » paraissent désormais tabous. Il y a donc une inflexion dans l'approche et l'analyse, sinon dans le « dicible ».

Cette critique de l'Etat était pourtant particulièrement développée de la part du PCF dans les années 1970. Comme le souligne Yves Roucaute, dans un livre de 1979, lors du $20^{\text {eme }}$ congrès du PCF, en 1970, G. Marchais déclarait que l'Etat constitue « une monstrueuse machine bureaucratique, dispendieuse et inefficace pour la nation, lointaine et tracassière pour la population $\gg^{14}$ (il est vrai qu'il s'agissait d'un Etat "giscardien » qui avait succédé à un «Etat UDR »). Compte tenu de cette situation, le PCF préconisait une limitation du rôle de l'administration et une extension des pouvoirs de la société civile. Ce faisant, il s'agissait de renverser le rapport entre l'Etat et les travailleurs. Le PCF se ralliait ainsi à idée d'autogestion ou de «participation» à appliquer au fonctionnement de la bureaucratie. Ces principes devaient s'incarner, par exemple, dans la mise en place de comités d'usagers. Etonnamment, la direction du PCF aujourd'hui semblent bien plus réservée (sinon muette) sur ce sujet (les agents des services publics et leurs organisations syndicales sont il est vrai rétifs à cette association des « usagers », tout en préférant ce terme à celui de clients). Au fond, la direction du PCF apparaît manquer de confiance dans la société civile ou fait le constat de son délitement, ce qui nécessite le développement de «services publics», vue comme des garanties ou tuteurs en même temps que nouvelles conquêtes.

Déjà à la Libération, devenu ministre d'Etat, chargé de la fonction publique, Maurice Thorez affirmait vouloir « refondre, rajeunir, démocratiser» l'administration. Il s'agissait d'en finir avec des « vices » - quasi-séculaires - de l'administration. Et de dénoncer aussi les « insuffisances », les «lenteurs», le «formalisme routinier et parfois tracassier de l'administration française «en dépit - ajoutait le secrétaire général du PCF - de ses effectifs pléthoriques $»^{15}$. Evidemment, ce type de critique n'apparaît plus de mise aujourd'hui de la part des dirigeants du PCF. Il importe d'abord de protéger et de développer les «services publics »... et la bureaucratie, quelle qu'elle soit, n'est plus nommée.

$\mathrm{Au}$ sortir de la seconde guerre mondiale, $\mathrm{M}$. Thorez écrivait encore : « Les fonctionnaires sont les premiers à déplorer les défauts de l'administration. Ils sont les premiers à en souffrir... Il en résulte une lente dévalorisation sur le plan matériel, comme sur le plan

\footnotetext{
G. Marchais (1980, p. 67-68).

Ibid., p. 103-104.

Cité par Y. Roucaute (1979, p. 98).

Ibid., p. 118
} 
moral $\gg^{16}$. Or, les dirigeants du PCF voulaient remédier à cette situation, par souci d'un bon fonctionnement l'Etat, car « il en [résultait] la désaffection [des] jeunes gens pour des emplois qui ne leur [assuraient] plus une vie décente et la considération qui doit s'attacher au bon serviteurs de l'Etat ». On pourrait trouver là des éléments pour une critique contemporaine de l'administration et, plus largement, des services publics. Mais la direction ou les militants du PCF privilégient aujourd'hui une critique externe de ceux-ci ou la mise en cause du contexte. En revanche, l'exercice des missions de service public, leur finalité, leur mode de gestion échappent à la critique, sauf à évoquer un mode de contrôle, qui reste assez flou, et associerait - mais comment ? - l'Etat, les élus et les citoyens. Et sauf à revendiquer également un développement de ces missions.

Enfin, comment Anicet Le Pors, dernier ministre communiste chargé de la fonction publique et des réformes administratives (1981-1984) aborde-t-il la question du service public ? Dans son livre, Contradictions, publié peu après son départ du gouvernement, il n'évoque guère cette notion, autre indicateur montrant que celle-ci n'avait pas encore ce caractère de leitmotiv politique qu'elle a acquise aujourd'hui dans le discours communiste. Pour A. Le Pors, le service public renvoie d'abord à quelques principes juridico-politiques. Il parle de son unité, principe d'autant plus important, selon lui, que le début des années 1980 voit la mise en œuvre de la décentralisation. L'unité du service public c'est en quelque sorte la garantie que - partout dans le territoire - le service sera bien effectué et effectué de la même manière (en dépit des «libertés » de la loi Deferre relative à la décentralisation). Autrement dit, la mise en œuvre de celle-ci ne signifie pas, même implicitement, de remise en cause du service public selon les territoires. Et cette unité (pour ne pas parler de garantie pour le jacobinisme), A. Le Pors en rappelle les fondements tenant aux particularités des missionnaires - soit des fonctionnaires - qui en assurent la charge (et inscrit dans le statut de la fonction publique qu'il contribue à réformer en 1983-1984) ${ }^{17}$ :

- principe d'égalité d'accès aux emplois publics

- principe d'indépendance de ces fonctionnaires vis-à-vis du pouvoir politique

- et, si le fonctionnaire bénéficie aussi d'une reconnaissance en tant que citoyen, dans le respect du pluralisme, la mission de service publique qu'il accomplit doit être neutre pour l'usager's.

Dans le discours communiste contemporain, tous ces aspects n'apparaissent plus discutés. Le service public - très présent, pas toujours bien défini, et d'abord notion générique - a pris une dimension plus idéologique. Il a comme remplacé, tout en l'adaptant, la dimension collectiviste qui n'est plus dicible dans le discours communiste.

Dès lors, il semble la clé, la solution, aux problèmes ou besoins multiples qui existent dans la société, d'autant plus importants, ou difficilement résolubles, que celle-ci est entrée en récession.

Le service public semble également prolonger de façon indéfinie l'Etat social et même réinventer un Etat producteur ou régulateur de l'économie'. Il ressortit aussi d'un projet idéologique, qui tend à réinventer plus ou moins implicitement le «communisme ». Il est enfin un «bien commun », ce qui lui confère un caractère quasi-sacré, en même temps qu'un rôle moteur de l'historicité. Cela étant, on ne peut que s'interroger sur le réalisme et le

\footnotetext{
"Ibid., p. 119.

"Voir Andolfatto (D.), «Les syndicats, freins ou moteurs du statut de la fonction publique », communication au colloque Les trente ans du statut général des fonctionnaires de l'Etat et des collectivités territoriales : Et après ?, colloque organisé par le Credespo, Université de Bourgogne, Dijon, 18-19 septembre 2014. Actes à paraître.

${ }^{18}$ A. Le Pors (1984, p. 52-53).

19 Voir Rosanvallon (Pierre), L'Etat en France : de 1789 à nos jours, Paris, Seuil, 1990.
} 
caractère - effectivement - opérationnel de ces perspectives. Autrement dit, pour le PCF, le service public a pris une sorte de dimension magique.

Bibliographie sélective :

Andolfatto (Dominique), PCF : de la mutation à la liquidation, Paris-Monaco, Le Rocher, 2005.

Blotin (Pierre), Communisme français. L’heure de vérité [brochure], 2006.

Buffet (Marie-George), Un peu de courage!, Entretien avec Kathleen Evin, Pierre Laurent, Bernard Coches, Michel Cool, Paris, Le cherche midi, 2004.

Buton (Philippe), Les lendemains qui déchantent. Le Parti communiste français à la Libération, paris, PFNSP, 1993.

Crozier (Michel), Le phénomène bureaucratique, Paris, Seuil, 1964.

Courtois (Stéphane), Marc Lazar, Histoire du Parti communiste français, Paris, PUF, 2000.

Furet (François), Le passé d'une illusion. Essai sur l'idée communiste au $X X^{\text {ine }}$ siècle, Paris, Calmann-Lévy et Robert Laffont, 1995.

Le Pors (Anicet), Contradictions. Entretiens avec Jean-Marie Colombani, Paris, Messidor, 1984.

Marchais (Georges), L’espoir au présent, Paris, Editions sociales, 1980.

PCF, Humanifeste du Parti communiste français à l'aube du siècle qui vient, Texte d'orientation adopté par le 36 $6^{\text {im }}$ congrès du PCF, 10 février 2013.

Roucaute (Yves), Le PCF et les sommets de l'Etat, Paris, PUF, 1979. 